\title{
Editorial
}

\section{Nanomaterials for Renewable Energy}

\author{
Shimou Chen, ${ }^{1}$ Liang $\mathrm{Li}^{2}{ }^{2}$ Hanwen Sun, ${ }^{3}$ Jian Sun, ${ }^{4}$ and Baowang $\mathrm{Lu}^{5}$ \\ ${ }^{1}$ Institute of Process Engineering, Chinese Academy of Sciences, Beijing 100190, China \\ ${ }^{2}$ Department of Physics, Jiangsu Key Laboratory of Thin Films, Soochow University, Suzhou 215006, China \\ ${ }^{3}$ College of Medicine and Nursing, Dezhou University, Dezhou 253023, China \\ ${ }^{4}$ Sandia National Laboratories, Livermore, CA 94550, USA \\ ${ }^{5}$ Graduate School of Environmental and Life Science, Okayama University, 3-1-1 Tsushima-naka, Kita-ku, \\ Okayama-shi 7008530, Japan
}

Correspondence should be addressed to Shimou Chen; chenshimou@ipe.ac.cn

Received 12 May 2015; Accepted 19 May 2015

Copyright (c) 2015 Shimou Chen et al. This is an open access article distributed under the Creative Commons Attribution License, which permits unrestricted use, distribution, and reproduction in any medium, provided the original work is properly cited.

With demand for sustainable energy, resource, and environment protection, new material technologies are constantly expanding during the last few couple of decades. An intensive attention has been given by the scientific communities. In particular, nanomaterials are increasingly playing an active role either by increasing the efficiency of the energy storage and conversion processes or by improving the device design and performance. This special issue presents recent research advances in various aspects of energy storage technologies, advanced batteries, fuel cells, solar cell, biofuels, and so on. Design and synthesis of novel materials have demonstrated great impact on the utilization of the sustainable energy, which need to solve the increasing shortage of resource and the issues of environmental pollution.

The special issue contains eight papers; the selected topic and the papers are not an exhaustive representation of nanomaterials for renewable energy applications. Nonetheless, they represent a broad range of knowledge on functional nanomaterials in chemistry, physics, biochemistry, materials science, engineering, and so forth, which are very helpful to share with the readers.

A review article titled "Mussel-Inspired Polydopamine Coated Iron Oxide Nanoparticles for Biomedical Application" is authored by X. Gu et al. In this review, the synthesis of iron oxide nanoparticles, the mechanism of dopamine selfoxidation, the interaction between iron oxide and dopamine, the functionality and the safety assessment of dopamine modified iron oxide nanoparticles, and the biomedical application of such nanoparticles are discussed.
The paper titled "A Green and Facile Synthesis of CarbonIncorporated $\mathrm{Co}_{3} \mathrm{O}_{4}$ Nanoparticles and Their Photocatalytic Activity for Hydrogen Evolution" is authored by L. Gao et al. They found that the carbon-incorporated $\mathrm{Co}_{3} \mathrm{O}_{4}$ nanoparticles were able to split pure water into hydrogen under visible light irradiation without any cocatalyst, which is mainly due to the enhanced light absorption behavior. This facile method provided a potential strategy for applying narrow bandgap semiconductors in pure water splitting.

The paper titled "Study of Phase Change Materials Applied to CPV Receivers" is authored by Z.-H. Shih et al. They focused on finding new materials to enhance the thermal dispreading and keep the temperature of solar cell as low as possible. They applied electric forward bias on solar cells to simulate the heat contributed from the concentrated sunlight and observed the thermal distribution of these three kinds of thermal spreading materials. Two levels of forward biases were chosen to test the samples and analyze the experiment results.

The paper titled "All-Polymer Solar Cells Based on Fully Conjugated Donor-Acceptor Block Copolymers with Poly(naphthalene bisimide) Acceptor Blocks: Device Performance and Thin Film Morphology" is authored by K. Nakabayashi et al. They fabricated all-polymer solar cells by using poly(3-hexylthiophene) (P3HT) and fully conjugated donor-acceptor (D-A) block copolymer (P3HT-PNBI$\mathrm{P} 3 \mathrm{HT}$ ) as donor and acceptor materials, respectively. Atomic force microscopy (AFM) and grazing incidence wide angle $\mathrm{X}$-ray scattering (GIWAXS) analyses reveal that device 
performance strongly depends on the P3HT:P3HT-PNBIP3HT thin film morphology. Their results suggest that P3HTPNBI-P3HT has the huge potentials for the usage as a nonfullerene acceptor material.

L. Wang and A. He synthesized polypropylene (PP)/clay nanocomposites by in situ intercalative polymerization with $\mathrm{TiCl}_{4} / \mathrm{MgCl}_{2} /$ clay compound catalyst. Their paper is titled "Microstructure and Thermal Properties of Polypropylene/Clay Nanocomposites with $\mathrm{TiCl}_{4} / \mathrm{MgCl}_{2} /$ Clay Compound Catalyst." In this paper, microstructure and thermal properties of PP/clay nanocomposites were studied by FTIR, XRD, TEM, TGA, and DSC, respectively, in detail. They found that clay layers in composites were exfoliated into nanometer size and dispersed uniformly in the PP matrix. The clay can enhance the thermal stability of PP materials efficiently.

W. Zhang et al. reported that when nano-MgO was prepared by the microwave-assisted method, the electric properties of its LDPE nanocomposite were obviously better than those of nano-MgO prepared by traditional heating method. Their paper titled "Influence of Nanocomposites of LDPE Doped with Nano-MgO by Different Preparing Methods on Its Dielectric Properties." In this paper, the influence of nano-MgO on several properties of LDPE nanocomposite was discussed, such as the space charge, volume resistivity as well as DC breakdown strength.

The paper titled "Theoretical Study on Cyclopeptides as the Nanocarriers for $\mathrm{Li}^{+}, \mathrm{Na}^{+}, \mathrm{K}^{+}$and $\mathrm{F}^{-}, \mathrm{Cl}^{-}, \mathrm{Br}^{-}$' is reported by $\mathrm{L}$. Liu and $\mathrm{S}$. Chen. The authors designed a series of experiments to compare the different capabilities of the different clyclopeptides in ions transport. The interaction process between a series of cyclopeptide compounds cyclo(gly) $n(n=4,6,8)$ and monovalent ions $\left(\mathrm{Li}^{+}, \mathrm{Na}^{+}, \mathrm{K}^{+}\right.$, $\mathrm{F}^{-}, \mathrm{Cl}^{-}$, and $\mathrm{Br}^{-}$) was studied using theoretical calculations. The mechanism of combination between the cyclo(gly)n and ions was discussed through binding energy, Mulliken electron population, and hydrogen bond.

Another theoretical paper titled " $A b$ Initio Theoretical Investigation on the Geometrical and Electronic Structures of Gallium Aurides: $\mathrm{GaAu}_{n}{ }^{0 /-}$ and $\mathrm{Ga}_{2} \mathrm{Au}_{n}{ }^{0 /-}(n=1-4)$ " is reported by W.-Z. Yao et al. They found that $\mathrm{GaAu}_{n}{ }^{0 /-}(n=$ $1-4)$ clusters with $n$-Au terminals and $\mathrm{Ga}_{2} \mathrm{Au}_{n}{ }^{0 /-}(n=1-4)$ clusters with bridged $\mathrm{Au}$ atoms possess geometric structures and bonding patterns similar to those of the corresponding gallium hydrides $\mathrm{GaH}_{n}{ }^{0 /-}$ and $\mathrm{Ga}_{2} \mathrm{H}_{n}{ }^{0 /-}$. In contrast to the highly symmetric ground states of $C_{2 v} \mathrm{Ga}_{2} \mathrm{Au}, \mathrm{C}_{2 v} \mathrm{Ga}_{2} \mathrm{Au}_{2}$, and $D_{3 h} \mathrm{Ga}_{2} \mathrm{Au}_{3}, C_{3 v} \mathrm{Ga}_{2} \mathrm{Au}_{4}$ is composed of strong interactions between a $\mathrm{Ga}^{+}$cation and the face of a tetrahedral $\mathrm{GaAu}_{4-}$ anion. The adiabatic and vertical detachment energies of the anions under study are calculated to facilitate their experimental characterization.

\section{Acknowledgments}

We would like to thank the authors who have submitted a manuscript to this special issue. Secondly, the fundamental work of all the reviewers of these papers is also very warmly acknowledged. Lastly, the lead editor thanks all the editors for their contribution in reviewing and assigning reviews for the submitted manuscripts.

Shimou Chen

Liang $\mathrm{Li}$

Hanwen Sun

Jian Sun

Baowang Lu 

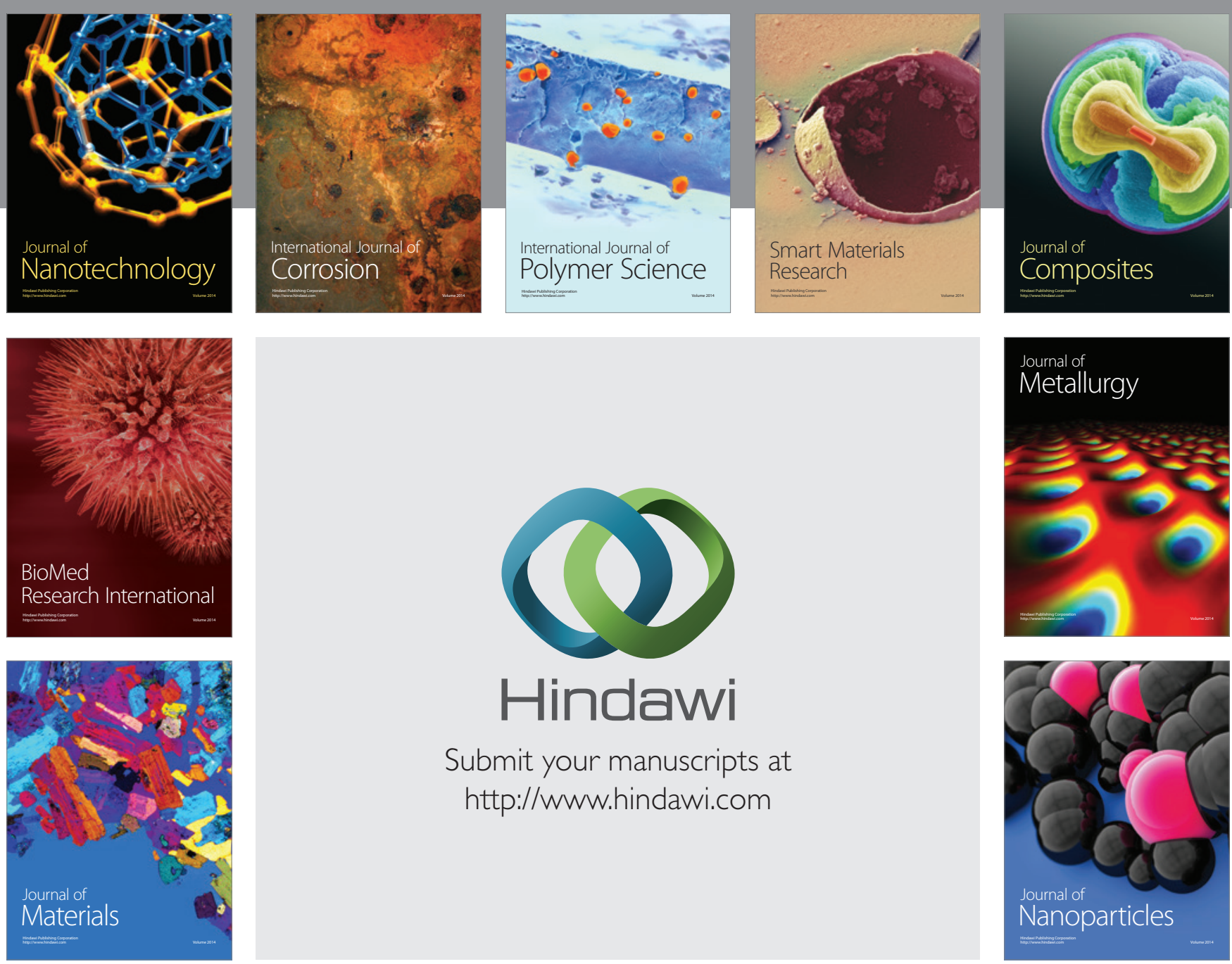

Submit your manuscripts at http://www.hindawi.com
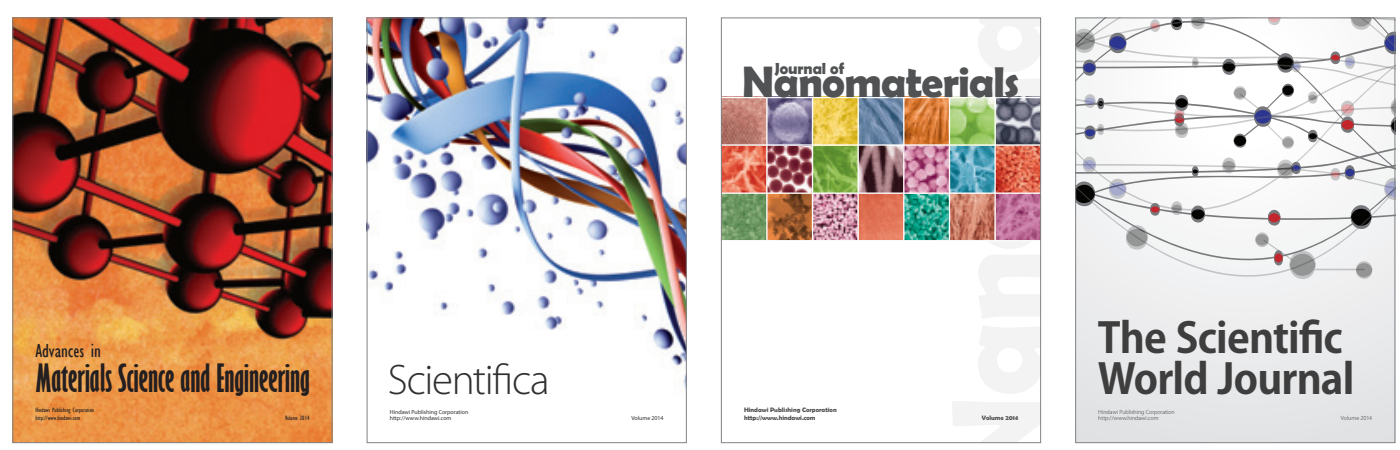

\section{The Scientific World Journal}
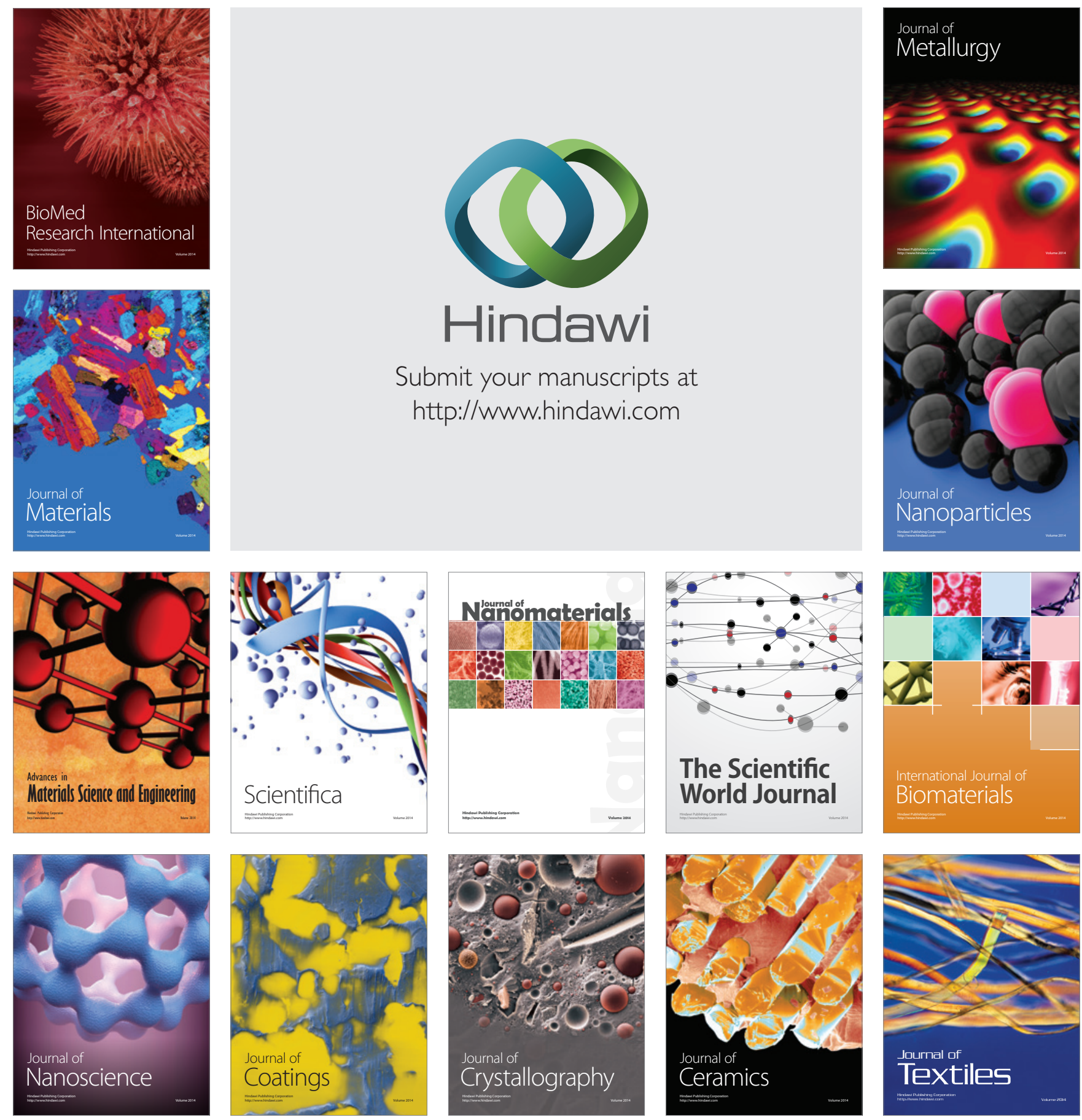